\title{
Cattle production potential in small, improved, mixed farms in Burundi
}

\author{
P Pozy 1, D Dehareng 2, AG Deswysen 3 \\ 1/institut des Sciences Agronomiques du Burundi, BP 795, Bujumbura ; 2 Faculté des Sciences Agronomiques, \\ Département des Productions Animales, Université du Burundi, BP 2940, Bujumbura, Burundi; \\ 3 Faculté des Sciences Agronomiques, Unité GENA, Université Catholique de Louvain, \\ Place Croix du Sud 2 (Boite 14), 1348 Louvain-la-Neuve, Belgique
}

Traditional mixed highland farms in Burundi show actually decreases in both foodcrops and livestock production, which are mainly due to decreasing soil fertility and overgrazing. A proposed technological way to obtain sustainable development of small subsistence farms is to better integrate vegetable and animal productions, for instance by realizing alternate and associated food and forage crops in the part of the farm which is usually devoted to forage.

Objective of this study is to evaluate cattle production potential of those small subsistence, however technologically improved, mixed farms in Burundi highlands.

Located on hilly landscape, 60 subsistence, technologically improved, mixed farms (mean : $0.86 \mathrm{ha}$ ) were monitored from 1985 to 1993. Those farmers grow foodcrops (beans, maize, sorgho, potato and banana) for their family nutrition (no cash crops) and manage cattle, mainly Sahiwal crossbred. Throughout the year, those cattle graze overday natural grass :
Eragrostis olivacea $K$. Schum on communal grazing areas. Overnight, they are housed indoors on each farm. During the wet season, they are fed forage produced by anti-erosive hedges ( $0.067 \mathrm{ha}$ ) composited by $85 \%$ Setaria $s p$ and $15 \%$ Calliandra calothyrsus. During the dry season, they are fed crop residues (bean haulms from 0.315 ha, sorgho stovers from $0.08 \mathrm{ha}$ ) and forage from alternate and associated food and forage fields (0.26 ha) composited by $2 / 3$ Tripsacum laxum and 1/3 Calliandra calothyrsus. Each farmer manage at least one cow $(330 \mathrm{~kg} ; 1,400 \mathrm{~kg}$ total milk production; $548 \mathrm{~d}$ calving interval for 175 recorded lactations) and its calf $(24 \mathrm{~kg}$ at birth) which is weaned at $120 \mathrm{~d}(60 \mathrm{~kg})$ and sold at $365 \mathrm{~d}(125 \mathrm{~kg}$; some female calves may be kept for replacement).

Annual mean total feedstuffs production on each farm allows to cover requirements of one dairy cow and its calf (with an excess of $4.6 \%$ UFL, $6.6 \%$ DM, $22.7 \%$ PDI) in addition to possible communal grazing production.

\begin{tabular}{|c|c|c|c|c|c|c|c|c|c|}
\hline \multirow[t]{2}{*}{ Feedstuffs } & \multicolumn{3}{|c|}{ Nutritive $\mathrm{v}$} & \multirow{2}{*}{$\begin{array}{c}\text { Area } \\
\text { ha }\end{array}$} & \multirow{2}{*}{$\begin{array}{c}\text { DM Yield } \\
\mathrm{kg} / \mathrm{ha} / \text { year }\end{array}$} & \multirow{2}{*}{$\mathrm{kgDM}$} & \multicolumn{2}{|c|}{ Annual yield } & \multirow{2}{*}{ gPDIE } \\
\hline & UFL & $\overline{\mathrm{gPDIN}}$ & $\mathrm{gPD} \overline{\mathrm{DI}}$ & & & & UFL & gPDIN & \\
\hline$s p^{2}$ & 0.67 & 72 & 82 & 0.057 & 17281 & 984 & 659 & 70859 & 80701 \\
\hline alliandra calot & 0.42 & 147 & 113 & 0 . & & 38 & 16 & & 4262 \\
\hline ean haulms & 0.88 & 83 & 97 & 0.315 & 669 & 211 & 185 & 17491 & 20441 \\
\hline Sorgho stovers & 0.59 & 38 & 49 & 0.080 & 8800 & 704 & 415 & 26752 & 34496 \\
\hline Calliandra calothyrsus & 0.42 & 147 & 113 & 0.087 & 1608 & 139 & 59 & 20486 & 15748 \\
\hline \multirow[t]{2}{*}{ Tripsacum laxum } & 0.61 & 51 & 68 & 0.173 & 9114 & 1580 & 964 & 80568 & 107121 \\
\hline & & & & & $\begin{array}{l}\text { Total yield } \\
\text { uirements }{ }^{3} \\
\text { Difference }\end{array}$ & $\begin{array}{c}3656 \\
3428 \\
227\end{array}$ & $\begin{array}{c}2298 \\
2198 \\
101\end{array}$ & $\begin{array}{c}221700 \\
180738 \\
40962\end{array}$ & 26307 \\
\hline
\end{tabular}

1 Data from Pozy et al (1995, in present Symposium), except sorgho stovers (no available data) which were considered to be similar to maize stovers (INRA, 1978, Alimentation des ruminants, ed INRA, Versailles, $p$ 544). ${ }^{2}$ From anti-erosive hedges. ${ }^{3}$ Annual total requirements of one cow and its calf (INRA, 1978, p 285, 346-355 (for UFL and PDI) ; Rivière, 1991, Alimentation des ruminants domestiques en milieu tropical, ed IEMVT, Maisons-Alfort, p 120 (for DM)). 